\title{
Funcionamiento cognitivo en pacientes con trastorno por uso de alcohol que inician tratamiento ambulatorio de deshabituación alcohólica
}

\section{Cognitive functioning in patients with alcohol use disorder who start outpatient treatment}

\author{
Rocio Villa*, Ashikan Espandian**, Pilar A. Sáiz*,***,**********, Mónica Astals $* * * * * *$, \\ Joana K. Valencia******, Emilia Martínez-Santamaría**, Sandra Álvarez**, \\ María Paz García-Portilla*,***,****,*****, Julio Bobes*,**************, Gerardo Flórez******. \\ * Servicio de Salud del Principado de Asturias (SESPA). España. ** Unidad de Conductas Adictivas, Complejo \\ Hospitalario Universitario de Ourense. España. *** Centro de Investigación Biomédica en Red de Salud Mental (CIBERSAM). \\ España. **** Área de Psiquiatría. Universidad de Oviedo. España. ***** Instituto de Investigación Sanitaria del Principado \\ de Asturias (ISPA). España. ****** Institut de Neuropsiquiatria i Addiccions, Institut Hospital del Mar \\ d'Investigacions Mèdiques (IMIM), Barcelona. España.
}

\section{Resumen}

El objetivo principal del presente estudio es analizar la presencia del deterioro cognitivo asociado al consumo de alcohol en los pacientes con trastorno por uso de alcohol moderado o grave que demandan tratamiento de deshabituación alcohólica ambulatorio. Para ello, se comparó una muestra de 111 pacientes con trastorno por uso de alcohol activo que iniciaban tratamiento ambulatorio versus 100 controles sanos. Se compararon variables sociodemográficas y clínicas asociadas al consumo de alcohol, como el craving de alcohol y la impulsividad. También se empleó en la comparación una batería sistematizada de pruebas cognitivas que permitía valorar las siguientes funciones: atención, memoria anterógrada, velocidad de procesamiento, fluidez verbal, función ejecutiva y actitud implícita ante las bebidas alcohólicas. En comparación con los controles sanos, los pacientes con trastorno por uso de alcohol moderado o grave presentaban un rendimiento significativamente inferior en todas las pruebas utilizadas, y por ello en todas las funciones cognitivas evaluadas, con la excepción de dos pruebas, el Iowa Gambling Test y el Implicit Association Test. El análisis a través de una matriz de correlaciones del grupo de pacientes indica que los pacientes que refieren más impulsividad y un consumo abusivo de alcohol más cronificado y con más adicción son los que presentan un mayor deterioro en su función cognitiva. El daño cognitivo asociado al consumo de alcohol se distribuyó de forma heterogénea entre los pacientes. El presente estudio confirma la presencia del deterioro cognitivo asociado al consumo de alcohol en los pacientes que demandan tratamiento ambulatorio. Palabras clave: Alcoholismo; Trastorno por uso de alcohol; Impulsividad; Daño cerebral asociado al consumo de alcohol; Función ejecutiva.

\begin{abstract}
The main objective of the present study is to analyze the presence of cognitive impairment associated with alcohol consumption in patients with moderate or severe alcohol use disorder seeking outpatient treatment for their dependence. To do this, we compared a sample of 111 patients with active alcohol use disorder who initiated ambulatory treatment with 100 healthy controls. We compared sociodemographic and clinical variables associated with alcohol consumption, such as alcohol craving and impulsivity. A systematized battery of cognitive tests was also used in the comparison, which allowed the evaluation of the following functions: Attention, anterograde memory, processing speed, verbal fluency, executive function and implicit attitude towards alcoholic beverages. Compared with healthy controls, patients with moderate or severe alcohol use disorder performed significantly worse in all tests used, and therefore in all cognitive functions evaluated, but for two tests, the Iowa Gambling Test and the Implicit Association Test. The analysis through a correlation matrix of the patient group indicates that patients who report more impulsivity and more chronic alcohol abuse and with more addiction are those who suffer greater deterioration in their cognitive function. Cognitive damage associated with alcohol consumption was distributed heterogeneously among patients. The present study confirms the presence of cognitive deterioration associated with alcohol consumption in patients seeking outpatient treatment.

Keywords: Alcoholism; Alcohol use disorder; Impulsivity; Alcohol related brain damage; Executive function.
\end{abstract}


$\mathrm{N}$ o hay duda de que el consumo crónico abusivo de alcohol daña el tejido cerebral y con ello deteriora la función cognitiva (Draper, Karmel, Gibson, Peut y Anderson, 2011; Erdozain et al., 2014; Florez, Espandian, Villa y Saiz, 2019; Hayes, Demirkol, Ridley, Withall y Draper, 2016; Laramee et al., 2015; Ridley, Draper y Withall, 2013; Sachdeva, Chandra, Choudhary, Dayal y Anand, 2016; Stavro, Pelletier y Potvin, 2013; Wollenweber et al., 2014). Este Daño Cerebral Asociado al Consumo de Alcohol (sus siglas en inglés son ARBD: Alcohol-Related Brain Damage) tiene como origen dos mecanismos tóxicos que actúan de forma combinada (Moretti, Caruso, Dal Ben, Gazzin y Tiribelli, 2017): por un lado, el efecto neurotóxico directo del etanol, mediado, principalmente, por una excitotoxicidad glutamatérgica (Stavro et al., 2013; Wollenweber et al., 2014); y, por otro lado, el daño asociado al déficit de tiamina que da lugar al Síndrome de Wernicke / Korsakoff (Galvin et al., 2010; Maharasingam, Macniven y Mason, 2013; Stavro et al., 2013). Estos dos mecanismos se combinan en los pacientes que presentan ARBD de una forma dimensional, es decir, el daño cerebral, y con ello el deterioro cognitivo que producen, puede ser desde leve hasta entrar en el rango de demencia (Moretti et al., 2017; Ridley et al., 2013; Zahr y Pfefferbaum, 2017). La presencia del ARBD está muy extendida entre los pacientes con trastorno por uso de alcohol, detectándose hasta en un $78 \%$ de las autopsias realizadas a estos pacientes (Ridley et al., 2013). El ARBD se caracteriza por una marcada atrofia cerebral generalizada producida por una destrucción neuronal y un daño en la sustancia blanca (Ridley et al., 2013; Stavro et al., 2013). Las siguientes áreas cerebrales parecen estar especialmente comprometidas en el ARBD (Zahr y Pfefferbaum, 2017): la sustancia blanca del Córtex Prefrontal, el Cuerpo Calloso y el Cerebelo; y la sustancia gris en el Córtex Prefrontal, el Hipotálamo y el Cerebelo.

Este daño cerebral trae consigo la presencia de alteraciones especialmente intensas en las siguientes funciones cognitivas (Aharonovich et al., 2018; Hagen et al., 2016; Hayes et al., 2016; Horton, Duffy, Hollins Martin y Martin, 2015; Maharasingam et al., 2013): memoria anterógrada, función ejecutiva (toma de decisiones, orientación temporal, juicios emocionales y fluencia verbal) y tareas visuoespaciales. La memoria de trabajo y el tiempo de latencia están generalmente alterados. En cada paciente el ARBD se presentará con un perfil de deterioro cognitivo diferente en intensidad y extensión en función distintas variables. Lo fundamental es la duración e intensidad del consumo de alcohol, especialmente los episodios de Binge Drinking, que darán lugar a una combinación específica de daño directo y déficit de tiamina (Golpe, Isorna, Barreiro, Brana y Rial, 2017; Hagen et al., 2016; Hayes et al., 2016; Horton et al., 2015). Pero la manifestación de este deterioro cognitivo también se verá modulado por otras variables (Hayes et al., 2016; Ridley et al., 2013; Sachdeva et al., 2016): las mujeres son más vulnerables a los efectos neurotóxicos del etanol, el nivel educativo alcanzado contrarresta el deterioro, la presencia de otros trastornos psiquiátricos (algunos, como la depresión, con un alta comorbilidad con el trastorno por uso de alcohol (Briere, Rohde, Seeley, Klein y Lewinsohn, 2014; Shoval et al., 2014)), el consumo de otros tóxicos y el daño vascular o el traumatológico empeoran el deterioro.

Es importante destacar que la afectación que el ARBD produce en las áreas cerebrales que controlan los impulsos, la atención y la memoria implicará que estos pacientes sean más vulnerables a la adicción al alcohol al ser menos capaces de controlar el impulso de beber alcohol pese a sus consecuencias negativas (Carmona-Perera, Sumarroca-Hernandez, Santolaria-Rossell, Perez-Garcia y Reyes Del Paso, 2019; Koob, 2003; Koob y Volkow, 2010; Mujica-Parodi, Carlson, Cha y Rubin, 2014; Volkow, Koob y McLellan, 2016)

En conjunto, el deterioro cognitivo del ARBD, y sus implicaciones en las actividades diarias de los pacientes afectados, es menor que el que producen los procesos degenerativos o de daño vascular en el cerebro, sobre todo a nivel del lenguaje (Horton et al., 2015). Además, el deterioro se detiene con la abstinencia, e incluso se revierte de forma parcial, de nuevo de forma variable para cada paciente, si dicha abstinencia se consolida. Se estima que se precisa al menos de un año de abstinencia para consolidar la mejoría, siendo la afectación en la memoria anterógrada la más resistente a la mejoría (Sachdeva et al., 2016).

Pese a la alta prevalencia del ARBD los estudios realizados hasta la fecha indican que tanto los servicios de Atención Primaria como las unidades específicas de atención a pacientes con trastorno por uso de alcohol no tienen por rutina valorar el deterioro cognitivo asociado al consumo de alcohol (Aharonovich et al., 2018; Draper et al., 2011; Hagen et al., 2016; Rehm et al., 2015a; Rehm et al., 2015b; Rehm, Rehm, Shield, Gmel y Gual, 2013; Rehm, Shield, Gmel, Rehm y Frick, 2013). Este escenario clínico es especialmente preocupante ya que las funciones cognitivas afectadas son de vital importancia a la hora de que el paciente pueda completar con éxito una desintoxicación / deshabituación alcohólica (Litten et al., 2015). Si la atención, la memoria, y la capacidad de planificación se viesen afectadas sería difícil que el paciente en tratamiento ambulatorio pueda seguir las directrices médicas y terapéuticas sin apoyo. Además, la mejoría significativa en estas funciones cognitivas tarda en producirse y no estará presente en los momentos iniciales críticos para consolidar la abstinencia (Sachdeva et al., 2016).

El objetivo del presente estudio es mejorar el conocimiento que en la actualidad hay sobre las alteraciones cognitivas que presentan los pacientes con trastorno por uso de alcohol que acuden demandando tratamiento para 
dejar de consumirlo. Para ello un grupo de estos pacientes será comparado con otro de población normativa sin problemas con el alcohol. La hipótesis principal del presente estudio es que el grupo de pacientes manifestará disfunciones cognitivas severas al compararse con el grupo control que disminuyen las posibilidades de éxito de un programa de deshabituación alcohólica ambulatorio.

\section{Material y Métodos}

\section{Participantes}

La muestra final estaba compuesta por 100 controles sanos y 111 pacientes con trastorno por uso de alcohol activo en el momento del reclutamiento. Los participantes fueron reclutados en tres dispositivos sanitarios: el Centro de Salud Mental La Calzada de Gijón (Área de Salud V de Asturias), la Unidad de Conductas Adictivas, Servicio de Psiquiatría del Complejo Hospitalario de Ourense y el Instituto de NeuroPsiquiatría y Adicciones, Parc de Salut Mar, Barcelona.

Los criterios de inclusión para los pacientes fueron: ser mayor de 18 años; cumplir criterios DSM-5 de trastorno por uso de alcohol moderado o grave, presentar a lo largo del último mes un consumo de alcohol superior a 60 gramos de etanol por día en varones y a 40 gramos de etanol por día en mujeres; expresar un claro deseo de controlar su consumo de alcohol; sin historia de tentativas suicidas y sin historia de Episodios Depresivos (uni o bipolares), con una puntuación en la Hamilton Depresion Rating Scale (HMDRS) en el momento de la evaluación inferior a 5; aceptar participar en el estudio y firmar el correspondiente consentimiento informado.

Los criterios de inclusión para el grupo control fueron: ser mayor de 18 años; no tener historia personal de ningún trastorno mental; no tener antecedentes familiares de trastorno por uso de alcohol, depresión mayor y/o tentativa suicida / suicidio consumado; presentar a lo largo del último mes un consumo de alcohol no superior a 30 gramos de etanol por día; aceptar participar en el estudio y firmar el correspondiente consentimiento informado.

Los criterios de exclusión para ambos grupos fueron: ser menor de 18 años; presentar patología orgánica o psiquiátrica (según el DSM-5) que a juicio de los investigadores impida la participación en el presente estudio, esto incluye los trastornos por uso de sustancias con la excepción del alcohol en el grupo de pacientes y el tabaco para ambos grupos; no aceptar participar en el estudio y firmar el correspondiente consentimiento informado. Investigadores cualificados entrevistaron a todos los candidatos a participar en el estudio para comprobar que cumpliesen los criterios de inclusión y exclusión.

Los controles se obtuvieron a través del personal de los centros y de los acompañantes de los pacientes. Fueron emparejados a los pacientes según criterios sociodemográficos.
Todos los participantes fueron informados exhaustivamente acerca de la naturaleza y características del estudio y dieron su consentimiento a participar por escrito. A todos ellos se les entregó una tarjeta regalo de 50 euros por su participación en el estudio. El estudio se aplicó siguiendo las directrices éticas y legales sobre la protección de datos personales y estudios con humanos, cumpliendo las directrices de la Declaración de Helsinki (Rickham, 1964). El estudio fue aprobado por los siguientes Comités de Ética de la Investigación: Pontevedra - Vigo - Ourense (2016313), Principado de Asturias (2017-06), y Parc de Salut Mar $(2017 / 7221 / \mathrm{I})$.

\section{Procedimiento}

El diseño es transversal con una comparativa caso - control. El primer paso fue la evaluación de todos los participantes para comprobar que cumplieran los criterios de inclusión y exclusión. A continuación, se recogieron las variables sociodemográficas y clínicas. Finalmente se procedió a cumplimentar la batería de pruebas cognitivas. Para completar todo el protocolo se utilizó una media de 3 sesiones no distanciadas entre si más de 72 horas.

\section{Variables}

A través de un cuestionario ad-hoc se recogieron las siguientes variables sociodemográficas y de consumo: sexo, edad, estado civil, convivencia, nivel educativo, situación laboral, edad de inicio del consumo de alcohol y tabaco, consume de alcohol y tabaco durante el último mes, edad de inicio de la dependencia al alcohol (casos), historia familiar de alcoholismo.

Variables clínicas recogidas a través de cuestionarios: para valorar la presencia de depresión como criterio de exclusión se usó la Hamilton Depression Rating Scale-17 items (HDRS-17) (Bech, 1990); para valorar la impulsividad se utilizó la Barratt Impulsiveness Scale - 11 (BIS-11) (Patton, Stanford y Barratt, 1995); y para valorar el Trastorno por uso de Alcohol se recurrió a la Obsessive Compulsive Drinking Scale (OCDS) (Anton, 2000).

La batería cognitiva utilizada para obtener las variables neuropsicológicas se recoge en la Tabla 1. Las pruebas utilizadas fueron: para medir el Cociente Intelectual (CI) global las pruebas del WAIS-III Búsqueda de Símbolos y Aritmética (Hagen et al., 2016); para medir la Atención el Test de Atención D2 (Steinborn, Langner, Flehmig y Huestegge, 2018); para medir la Memoria el California Verbal Learning Test (CVLT) (Elwood, 1995) y las pruebas del WAIS Clave de Números y Amplitud de Memoria de Dígitos (Hagen et al., 2016); para medir la Función Ejecutiva el FAS y categoría semántica de anímales (del Ser Quijano et al., 2004), el Test de Stroop (SCWT) (Scarpina y Tagini, 2017), el Wisconsin Card Sorting Test (WCST) (Nyhus y Barcelo, 2009), y el Iowa Gambling Test (IGT) (Steingroever, Wetzels, Horstmann, Neumann y Wagenmakers, 
Tabla 1. Batería de pruebas neuropsicológicas.

\begin{tabular}{|c|c|c|}
\hline Prueba neuropsicológica & Función principal evaluada & Características \\
\hline Busqueda de símbolos (del WAIS-III) & Velocidad de procesamiento $(\mathbf{C l})$ & $\begin{array}{l}\text { Mide la capacidad de identificar con rapidez la } \\
\text { presencia de figuras en una serie. No verbal. }\end{array}$ \\
\hline Aritmética (del WAIS-III) & Razonamiento abstracto $(\mathbf{C l})$ & $\begin{array}{l}\text { Mide la resolución mental de problemas } \\
\text { aritméticos en tiempo límite. Verbal. }\end{array}$ \\
\hline Test de Atención D2 & $\begin{array}{l}\text { Atención sostenida / inhibición de la } \\
\text { respuesta (Atención) }\end{array}$ & $\begin{array}{l}\text { Mide la habilidad para centrarse en estímulos visuales } \\
\text { relevantes e ignorar los irrelevantes. No verbal. }\end{array}$ \\
\hline California Verbal Learning Test (CVLT) & $\begin{array}{l}\text { Recuerdo inmediato, diferido e identificación } \\
\text { (Memoria) }\end{array}$ & $\begin{array}{l}\text { Mide la capacidad para recordar listas de palabras a lo } \\
\text { largo de varios intentos, sin y con interferencias. Verbal. }\end{array}$ \\
\hline Clave de números (del WAIS-III) & Memoria de trabajo (Memoria) & $\begin{array}{l}\text { Mide la rapidez para convertir números en símbolos } \\
\text { según una secuencia establecida. No verbal. }\end{array}$ \\
\hline Amplitud de memoria de dígitos (del WAIS-III) & Memoria a corta plazo (Memoria) & $\begin{array}{l}\text { Mide la capacidad para recordar y seguir } \\
\text { una secuencia de números. Verbal. }\end{array}$ \\
\hline FAS y categoría semántica de animales & Fluidez verbal (Función ejecutiva) & $\begin{array}{l}\text { Mide la capacidad para generar listas de } \\
\text { palabra por categorías. Verbal. }\end{array}$ \\
\hline Test de Stroop (SCWT) & $\begin{array}{l}\text { Atención dividida y resistencia ante } \\
\text { interferencias (Función ejecutiva) }\end{array}$ & $\begin{array}{l}\text { Mide la capacidad para el reconocimiento } \\
\text { de colores. No verbal. }\end{array}$ \\
\hline Wisconsin Card Sorting Test (WCST) & $\begin{array}{l}\text { Razonamiento abstracto y flexibilidad } \\
\text { cognitiva (Función ejecutiva) }\end{array}$ & $\begin{array}{l}\text { Mide la capacidad de selección de tarjetas } \\
\text { en función de categorías. No verbal. }\end{array}$ \\
\hline Iowa Gambling Test (IGT) & $\begin{array}{l}\text { Toma de decisiones y flexibilidad cognitiva } \\
\text { (Función ejecutiva) }\end{array}$ & $\begin{array}{l}\text { Mide la capacidad de selección de estímulos en función } \\
\text { de recompensas a corto y largo plazo. No verbal. }\end{array}$ \\
\hline Implicit Association Test (IAT) & $\begin{array}{l}\text { Actitud implícita ante un estímulo } \\
\text { (Procesamiento automático) }\end{array}$ & $\begin{array}{l}\text { Mide la rapidez a la hora de emparejar } \\
\text { palabras en función de actitudes implícitas } \\
\text { relacionadas con el alcohol. No verbal. }\end{array}$ \\
\hline
\end{tabular}

2013); y para medir el Procesamiento Automático el Implicit Association Test (IAT) adaptado para el consumo de alcohol (Ostafin, Marlatt y Greenwald, 2008).

\section{Análisis de datos}

Las comparaciones de las variables continuas entre los dos grupos objeto de estudio se realizó por medio del test t de Student, mientras que el análisis de las diferencias existentes en la distribución de las variables categóricas entre ambos grupos se efectuó por medio del test de Chi-cuadrado. También se compararon las variables clínicas y las cognitivas mediante una matriz de correlaciones. Se escogió un nivel de significancia de $\mathrm{p}<0.05$.

\section{Resultados}

La Tabla 2 muestra la distribución de las variables sociodemográficas y clínicas de la muestra, indicando en que variables hay diferencias significativas entre los pacientes y los controles sanos.

\section{Variables Sociodemográficas (Tabla 2)}

No existen diferencias significativas con respecto a las variables que pueden ejercer un sesgo en lo que a la valoración cognitiva y el deterioro cognitivo asociado al consumo de alcohol se refiere: edad, sexo y años de estudios completados. Sin embargo, el trastorno por uso de alcohol sí impli- ca la presencia de diferencias significativas con respecto a las siguientes variables clínicas y sociodemográficas: una mayor frecuencia entre los controles a tener pareja $\left(\mathrm{X}^{2}=4,48, \mathrm{p}=\right.$ $0,035)$, y a vivir con algún familiar $\left(\mathrm{X}^{2}=12,385, \mathrm{p}=0,002\right), \mathrm{y}$ a estar activos laboralmente $\left(\mathrm{X}^{2}=36,828, \mathrm{p}<0,001\right)$.

\section{Variables relacionadas con el consumo de alcohol, de tabaco y con la impulsividad (Tabla 2)}

EL trastorno por uso de alcohol implicaba la presencia de diferencias significativas en lo referente a las siguientes variables: mayor impulsividad medida a través de la BIS (BIS-11 cognitivo $(\mathrm{t}=-3,60, \mathrm{p}<0,001)$, BIS-11 motor $(\mathrm{t}=-3,02, \mathrm{p}=0,003)$, BIS-11 no planificado $(\mathrm{t}=-3,35, \mathrm{p}$ $=0,001)$, BIS-11 total $(\mathrm{t}=-4,04, \mathrm{p}<0,001))$, un consumo de alcohol más patológico medido con la OCDS ( OCDS - Obsesivo ( $\mathrm{t}=-14,18, \mathrm{p}<0,001)$, OCDS - Impulsivo $(\mathrm{t}=$ $-22,95, \mathrm{p}<0,001)$, OCDS - Total $(\mathrm{t}=-21,23, \mathrm{p}<0,001)$, una edad más precoz en el inicio del consumo de tabaco $(\mathrm{t}=$ $3,96, p<0,001)$ y un mayor consumo de cigarrillos por día $(\mathrm{t}=-5,10, \mathrm{p}<0,001)$, un mayor consumo de alcohol por día (UBEs) ( $\mathrm{t}=-14,8, \mathrm{p}<0,001)$, un mayor número de familiares afectados por el consumo de alcohol $(\mathrm{t}=-4,73, \mathrm{p}$ $<0,001)$.

\section{Variables cognitivas (Tabla 3 )}

En la Tabla 3 se presentan los resultados obtenidos en cada grupo con respecto a las variables neuropsicológicas 
Tabla 2. Variables sociodemográficas y relacionadas con el consumo de alcohol.

\begin{tabular}{|c|c|c|c|c|c|c|c|}
\hline & \multicolumn{2}{|c|}{ Controles (100) } & \multicolumn{2}{|c|}{ Casos (111) } & \multicolumn{2}{|c|}{ Total (211) } & \multirow[b]{2}{*}{ p } \\
\hline & media & DS & media & DS & media & DS & \\
\hline Edad & 48,66 & 9,569 & 49,07 & 8,405 & 48,88 & 8,956 & 0,741 \\
\hline Edad inicio de consumo de alcohol & 18,21 & 6,414 & 17,21 & 4,226 & 17,56 & 5,114 & 0,274 \\
\hline UBEs día durante el último mes & 0,53 & 0,688 & 9,613 & 6,426 & 5,308 & 6,521 & $<0,001$ \\
\hline Edad de inicio de consumo de nicotina & 18,1 & 4,687 & 14,16 & 7,541 & 15,42 & 6,996 & $<0,001$ \\
\hline Cigarrillos fumados por día durante el último mes & 3,75 & 6,722 & 9,811 & 10,338 & 6,938 & 9,3 & $<0,001$ \\
\hline Años de estudios completados & 12,76 & 2,417 & 13,3 & 2,881 & 13,04 & 2,679 & 0,142 \\
\hline Familiares afectados por el alcohol & 0,27 & 0,566 & 0,973 & 1,449 & 0,6398 & 1,172 & $<0,001$ \\
\hline BIS11- Cognitivo & 14,85 & 5,809 & 18,58 & 9,038 & 16,81 & 7,885 & $<0,001$ \\
\hline BIS11- Motor & 13,82 & 6,327 & 16,59 & 7,03 & 15,28 & 6,833 & 0,003 \\
\hline BIS11- No planificado & 16,28 & 6,482 & 19,72 & 8,379 & 18,09 & 7,717 & 0,001 \\
\hline BIS-Total & 44,99 & 15,54 & 55,09 & 20,67 & 50,3 & 19,06 & $<0,001$ \\
\hline OCDS- Obsesivo & 0,06 & 0,371 & 6,5135 & 4,78 & 3,455 & 4,74 & $<0,001$ \\
\hline OCDS-Impulsivo & 0,72 & 1,349 & 10,703 & 4,356 & 5,972 & 5,98 & $<0,001$ \\
\hline OCDS-Total & 0,79 & 1,559 & 17,207 & 7,981 & 9,427 & 10,101 & $<0,001$ \\
\hline Edad de inicia de la dependencia al alcohol & & & 33,362 & 9,148 & & & \\
\hline GOT & & & 38,53 & 25,44 & & & \\
\hline GPT & & & 37,02 & 20,37 & & & \\
\hline GGT & & & 130,4 & 167,5 & & & \\
\hline VCM & & & 95 & 6,363 & & & \\
\hline Sexo (\%varones) & & $74 \%$ & & $78,37 \%$ & & $76,30 \%$ & 0,4551 \\
\hline Estado civil (\%casados - pareja de hecho) & & $56 \%$ & & $41,44 \%$ & & $48,34 \%$ & 0,035 \\
\hline Convivencia (\% solo) & & $20 \%$ & & $24,32 \%$ & & $22,27 \%$ & 0,002 \\
\hline Situación laboral (\%activos) & & $80 \%$ & & $39,63 \%$ & & $58,76 \%$ & $<0,001$ \\
\hline
\end{tabular}

Nota. DS: Desviación estándar; UBE: Unidad de Bebida Estandar; BIS: Barratt Impulsiveness Scale; OCDS: Obsessive Compulsive Drinking Scale; GOT: Glutamano Oxalacetato Transaminasa; GPT: Glutamano Piruvato Transaminasa; GGT: Gamma Glutamil Transferasa; VCM: Volumen Corpuscular Medio.

obtenidas a través de las distintas pruebas cognitivas realizadas. En todas las pruebas se obtuvieron resultados significativos que indicaban una mejor función cognitiva en el grupo control, con la excepción del IGT y del IAT, en estas dos pruebas no se encontraron diferencias significativas entre ambos grupos.

También se realizó un estudio de correlaciones para estudiar cómo influyen las variables relacionadas con el consumo de alcohol y la impulsividad con las pruebas cognitivas en el grupo de pacientes. En la tabla 4 se presentan las correlaciones que fueron significativas. Se observó que las variables que se relacionan con el consumo crónico de alcohol (Años de dependencia al alcohol, Porcentaje de vida con Dependencia al Alcohol, OCDS y BIS) correlacionaban de forma más intensa con una peor función cognitiva en comparación con las variables que se relacionan con el consumo agudo de alcohol (UBEs, GOT,GPT, GGT y VCM).
En la Tabla 5 se presenta una matriz de correlaciones entre las variables relacionadas con el consumo de alcohol y la impulsividad en la valoración basal de los pacientes. Se observó una correlación positiva significativa entre el BIS y el OCDS, y entre las variables analíticas relacionadas con el consumo de alcohol (GOT,GPT,GGT y VCM) y con las UBEs.

\section{Discusión}

En el presente estudio, se comparó el rendimiento cognitivo, utilizando una batería sistematizada de pruebas verbales y no verbales, de un grupo de pacientes con trastorno por uso de alcohol que demandan tratamiento de deshabituación frente a un grupo de voluntarios sanos, emparejados para las principales variables sociodemográficas que influyen en la capacidad cognitiva (edad, sexo y años de estudios completados). Tal y como era de esperar, y confir- 
Tabla 3. Comparación de las pruebas neuropsicológicas entre los controles y los pacientes en la valoración basal.

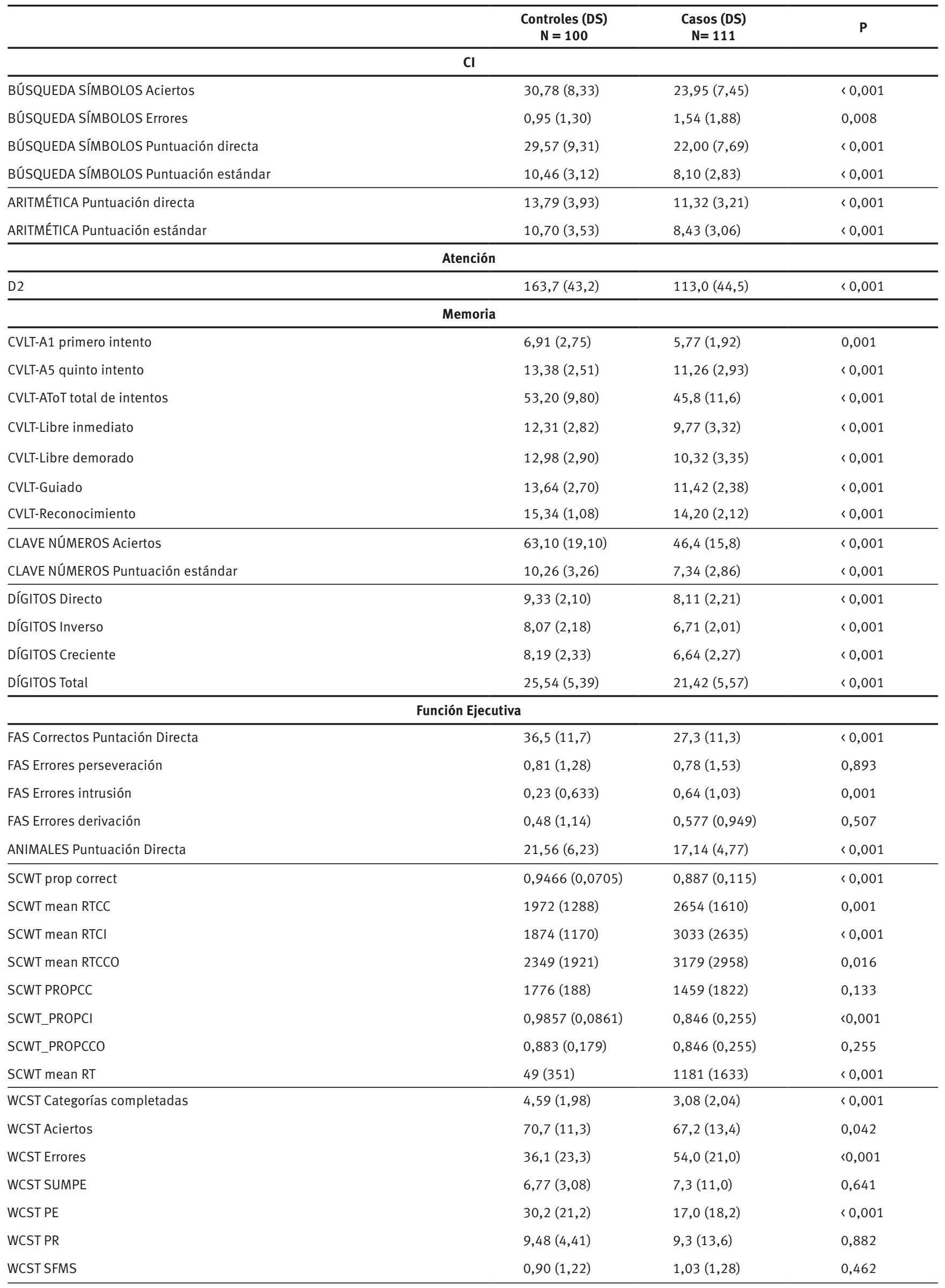




\begin{tabular}{|c|c|c|c|}
\hline \multicolumn{4}{|l|}{ WCST } \\
\hline TRIAL FIRST & $22,6(26,7)$ & $30,3(34,3)$ & 0,07 \\
\hline WSCT Cl & $18,4(16,8)$ & $22,6(19,4)$ & 0,096 \\
\hline WCST FI & $25,4(16,2)$ & $33,2(20,1)$ & 0,002 \\
\hline WSCT NI & $28,5(22,9)$ & $31,6(26,3)$ & 0,36 \\
\hline WSCT C2 & $15,8(15,3)$ & 32 (176) & 0,348 \\
\hline WSCT DIFFC1F1 & $-1315(13095)$ & $-9,5(30,3)$ & 0,321 \\
\hline WSCT DIFFF1N1 & $-1,7(28,3)$ & $1,5(36,4)$ & 0,466 \\
\hline WSCT DIFFN1C2 & $12,2(24,7)$ & $16,7(29,1)$ & 0,234 \\
\hline WSCT DIFFC2F2 & $-0,2(21,4)$ & $1,7(22,8)$ & 0,527 \\
\hline IGT Total & 2039 (964) & $1836(822)$ & 0,104 \\
\hline IGT CA & $49,9(16,1)$ & $46,5(15,5)$ & 0,125 \\
\hline IGT CDA & $50,1(16,1)$ & $53,5(15,5)$ & 0,125 \\
\hline $\begin{array}{l}\text { IGT NET } \\
5 \mathrm{AD}\end{array}$ & $10,56(4,85)$ & $9,72(4,60)$ & 0,2 \\
\hline $\begin{array}{l}\text { IGT NET } \\
5 \mathrm{DIS}\end{array}$ & $9,44(4,85)$ & $10,28(4,60)$ & 0,2 \\
\hline \multicolumn{4}{|c|}{ Procesamiento automático } \\
\hline IAT & $-0,569(0,515)$ & $-0,483(0,480)$ & 0,215 \\
\hline
\end{tabular}

mando la hipótesis principal, el grupo de pacientes presentó déficits significativos con respecto a los voluntarios sanos en casi la totalidad de las pruebas. Tanto la atención y la velocidad de procesamiento, como la memoria anterógrada y la de trabajo, como la función ejecutiva (fluidez verbal, resistencia a la interferencia, razonamiento abstracto y flexibilidad cognitiva) se encontraban alteradas de forma significativa en los pacientes. De hecho, sólo en dos pruebas, IGT e IAT, no se encontraron diferencias significativas. Estos hallazgos confirman los resultados obtenidos con anterioridad en estudios de neuroimagen y neuropatología que indican la presencia de un daño difuso en todo el cerebro, pero con afectación más severa del Córtex Prefrontal, el Hipotálamo y el Cerebelo (Erdozain et al., 2014; Hayes et al., 2016; Zahr y Pfefferbaum, 2017), y son similares a los obtenidos por otros estudios en los que se ha analizado la función cognitiva en pacientes con trastorno por uso de alcohol versus población control (Aharonovich et al., 2018; Romero-Martinez, Vitoria-Estruch y Moya-Albiol, 2020).

Como grupo, los pacientes eran conscientes de su mayor impulsividad e incapacidad para la planificación, tal y como reflejan los resultados obtenidos en el BIS-11. Además, estas alteraciones cognitivas, unidas a las consecuencias negativas que implica la intoxicación alcohólica, afectan a la capacidad de integración sociofamiliar de los pacientes. Los resultados indican que los pacientes tienden más a carecer de pareja estable, a vivir en soledad y a no estar activos laboralmente. Por si esto fuera poco, su salud física está más comprometida, no sólo debido al consumo abusivo de alcohol, sino también al presentar un consumo superior de tabaco, que ha estado presente durante más años. Cierto es que, en la adicción al tabaco de los pacientes con trastorno por uso de alcohol, no sólo la impulsividad y la falta de función ejecutiva ejercen un papel en el mantenimiento de la misma, otros factores genéticos, neurobiológicos y ambientales también ejercen un papel determinante, pero sin duda contribuyen de forma significativa (Koob, 2003; Koob y Volkow, 2010; Volkow et al., 2016).

No se observó que ninguna variable relacionada con el IGT permitiese discriminar entre pacientes y controles sanos. Aunque inicialmente diversos estudios encontraron diferencias significativas en esta prueba que mide funciones cognitivas como la flexibilidad cognitiva y la toma de decisiones, lo cierto es que el grueso de la investigación más reciente indica que esta prueba tiene poca capacidad discriminativa a la hora de distinguir a los pacientes con trastorno por uso de alcohol frente a los controles sanos (Hagen et al., 2016). Básicamente, la correcta realización de la prueba exige de una función cognitiva excelente. Es por esto, que los resultados que obtiene un grupo control representativo y bien pareado con el grupo de pacientes como el nuestro son mediocres. El análisis de las variables 
Tabla 4. Correlaciones significativas entre las variables relacionadas con el consumo de alcohol y la impulsividad y las variables cognitivas en el grupo de pacientes.

\begin{tabular}{|c|c|}
\hline $\begin{array}{l}\text { Variables consumo de alcohol / } \\
\text { impulsividad }\end{array}$ & Variables cognitivas con correlación significativa \\
\hline Años de dependencia al alcohol & $\begin{array}{l}\text { D2 }(-0,2, p=0,032) \\
\text { CVLT A5 }(-0,28, p=0,0029) \\
\text { CVLT AToT }(-0,23, p=0,013) \\
\text { CVLT Libre inmediato }(-0,27, P=0,004) \\
\text { CVLT Libre demorado }(-0,21, P=0,0027) \\
\text { Búsqueda de Símbolos Aciertos }(-0,31, P=0,0007) \\
\text { Búsqueda de Símbolos Puntuación total }(-0,28, P=0,0024) \\
\text { Aritmética Puntuación Estándar }(-0,25, P=0,0063) \\
\text { WCST SUMPE }(0,23, P=0,012) .\end{array}$ \\
\hline $\begin{array}{l}\text { Porcentaje de vida con } \\
\text { Dependencia al alcohol }\end{array}$ & $\begin{array}{l}\text { CVLT A5 }(-0,19, p=0,004) \\
\text { CVLT Libre inmediato }(-0,19, p=0,036) \\
\text { Búsqueda de Símbolos Aciertos }(-0,23, p=0,014) \\
\text { Búsqueda de Símbolos Puntuación Total }(-0,22, p=0,018) \text { Búsqueda de Símbolos Puntuación Estándar }(-0,19, p=0,046) \\
\text { WCST SUMPE }(0,19, p=0,036) \\
\text { IAT }(0,21, p=0,025)\end{array}$ \\
\hline UBEs & Aritmética Puntuación Estándar $(-0,20, p=0,033)$ \\
\hline GOT & No se encontró ninguna correlación significativa \\
\hline GPT & SWCT PROPCC $(-0,21, p=0,024)$ \\
\hline GGT & No se encontró ninguna correlación significativa \\
\hline VCM & $\begin{array}{l}\text { Aritmética Puntuación Directa }(-0,19, p=0,038) \\
\text { Aritmética Puntuación Estándar }(-0,20, p=0,0036)\end{array}$ \\
\hline OCDS-Obsesivo & $\begin{array}{l}\text { Animales Puntuación Directa }(0,18, p=0,049) \\
\text { WCST Aciertos }(-0,18, p=0,049)\end{array}$ \\
\hline OCDS-Impulsivo & $\begin{array}{l}\text { Búsqueda de Símbolos Puntuación Estándar }(-0,29, p=0,0019) \\
\text { WCST Categorías completadas }(-0,20, p=0,027) \\
\text { WCST Aciertos }(-0,26, p=0,0046) \\
\text { WCST Errores }(0,26, p=0,0048) \\
\text { WCST DIFF2N2 }(-0,22, p=0,019\end{array}$ \\
\hline OCDS-Total & $\begin{array}{l}\text { Búsqueda de Símbolos Puntuación Estándar }(-0,25, p=0,0075) \\
\text { IGT CA }(0,20, p=0,03) \\
\text { IGT CDA }(-0,26, p=0,03) \\
\text { WCST Aciertos }(-0,26, p=0,0054) \\
\text { WCST Errores }(0,25, p=0,0084) \\
\text { WCST DIFF2N2 }(-0,21, p=0,023) \\
\text { IAT }(0,18, p=0,048)\end{array}$ \\
\hline BIS-Cognitivo & $\begin{array}{l}\text { CVLT Guiado }(0,19, p=0,038) \\
\text { SCWT mean RTCC }(-0,29, P=0,001) \\
\text { SCWT mean RTCI }(-0,23, p=0,012) \\
\text { SCWT PROPCI }(0,23, p=0,012) \\
\text { SCWT mean RT }(-0,3, p=0,0012) \\
\text { FAS Correctos Puntuación Directa }(-0,2, p=0,032) \\
\text { Dígitos Inverso }(-0,24, p=0,009) \\
\text { Dígitos Creciente }(-0,31, p=0,0008) \\
\text { Dígitos Total }(-0,24, p=0,01) \\
\text { WCST Categorías completadas }(-0,31, p=0,0009) \\
\text { WCST Aciertos }(-0,39, p<0,001) \\
\text { WCST Errores }(0,36, p<0,001)\end{array}$ \\
\hline BIS-Motor & $\begin{array}{l}\text { SCWT mean RTCI }(-0,25, p=0,007) \\
\text { SCWT PROPCI }(0,19, p=0,042) \\
\text { SCWT mean RT }(-0,38, p<0,001) \\
\text { FAS Correctos Puntuación Directa }(-0,2, p=0,032) \\
\text { WCST Categorías completadas }(-0,24, p=0,0088) \\
\text { WCST Aciertos }(-0,26, p=0,0056) \\
\text { WCST Errores }(0,35, p=0,0065) \\
\text { WCST FI }(-0,3, p=0,0011) \\
\text { WCST DIFF1N1 }(-0,22, p=0,019)\end{array}$ \\
\hline BIS-No planificado & $\begin{array}{l}\text { SWCT mean RTCC }(-0,33, p=0,0004) \\
\text { SWCT mean RTCI }(-0,31, p=0,0008) \\
\text { SCWT PROPCI }(0,23 p=0,011) \\
\text { SCWT mean RT }(-0,38, p<0,001) \\
\text { Dígitos Creciente }(-0,18, p=0,047)\end{array}$ \\
\hline
\end{tabular}




\begin{tabular}{ll}
\hline & WCST Categorías completadas $(-0,38, p<0,001)$ \\
& WCST Aciertos $(-0,45, p<0,001)$ \\
& WCST Errores $(0,40, p<0,001)$ \\
& WCST C1 $(-0,25 p=0,008)$ \\
& WCST F1 $(-0,28 p=0,002)$ \\
& WCST C2 $(-0,2 p=0,033)$ \\
& WCST DIFF1N1 $(-0,23, p=0,019)$ \\
& WCST DIFF2N2 $(-0,18, p=0,0029)$ \\
& IAT $(0,20, p=0,047)$. \\
\hline BIS-Total & SCWT mean RTCC $(-0,31, p=0,0007)$ \\
& SWCT mean RTCI $(-0,30, p=0,001)$ \\
& SCWT mean RT $(-0,30, p<0,001$ \\
& FAS Correctos Puntuación Directa $(-0,21, p=0,024)$ \\
& Dígitos Inverso $(-0,19, p=0,044)$ \\
& Dígitos Creciente $(-0,25, p=0,0065)$ \\
& Dígitos Total $(-0,22, p=0,02)$ \\
& WCST Categorías completadas $(-0,36, p<0,001)$ \\
& WCST Aciertos $(-0,45, p<0,001)$ \\
& WCST Errores $(0,40, p<0,001)$ \\
& WCST C1 $(-0,20, p=0,0034)$ \\
& WCST F1 $(-0,27, p=0,003)$ \\
& WCST DIFFF1N1 $(-0,23, p=0,014)$ \\
& WCST DIFFF2N2 $(-0,18, p=0,047)$ \\
& IAT $(0,20, p=0,031)$ \\
\hline
\end{tabular}

Nota. UBE: Unidad de Bebida Estandar; BIS: Barratt Impulsiveness Scale; OCDS: Obsessive Compulsive Drinking Scale; GOT: Glutamano Oxalacetato Transaminasa; GPT: Glutamano Piruvato Transaminasa; GGT: Gamma Glutamil Transferasa; VCM: Volumen Corpuscular Medio; SCWT: prop correct: Proporción de respuestas totales correctas; mean RTCC: Latencia media de respuesta correctas congruentes; mean RTCI: Latencia media de respuestas correctas incongruentes; mean RTCCO: Latencia media de respuestas correctas; PROPCC: Proporción de respuestas correctas congruentes; PROPCI: Proporción de respuestas correctas incongruentes; PROPCCO: Proporción de respuestas correctas; mean RT: Latencia media de respuestas totales correctas; WCST: SUMPE: Suma de todos los ensayos incorrectos con errores; PE: Porcentaje de errores perseverativos; PR: Porcentaje de perseveraciones en la pruebas; SFMS: Número total de ocasiones en que se selecciona una carta incorrecta; TRIAL FIRST: Número de ensayos necesarios para completar la primera categoría después de hacer al menos 5 correctas; CI: Porcentaje de errores en la primera categoría de color ; NI: porcentaje de errores en la primera categoría de número; Fl: porcentaje de errores en la primera categoría de forma; C2: porcentaje de error en la segunda categoría de color; DIFF: Diferencia en los porcentajes de errores entre categorías adyacentes.

Tabla 5. Matriz de correlaciones entre las variables relacionadas con el consumo de alcohol y la impulsividad en la valoración basal de los pacientes.

\begin{tabular}{|c|c|c|c|c|c|c|c|c|c|c|c|c|c|c|}
\hline & Años & $\%$ & UBES & GOT & GPT & GGT & VCM & $\begin{array}{c}\text { BIS } 11 \\
\text { C }\end{array}$ & $\begin{array}{c}\text { BIS } 11 \\
M\end{array}$ & $\begin{array}{c}\text { BIS } 11 \\
\mathbf{N}\end{array}$ & $\begin{array}{c}\text { BIS } 11 \\
T\end{array}$ & $\begin{array}{c}\text { OCDS } \\
0\end{array}$ & $\begin{array}{c}\text { OCDS } \\
\text { C }\end{array}$ & $\begin{array}{c}\text { OCDS } \\
T\end{array}$ \\
\hline Años & 1 & $0,904^{\star \star}$ & $-0,034$ & $-0,016$ & $-0,057$ & 0,080 & $0,207^{\star}$ & $-0,158$ & $-0,183$ & 0,002 & $-0,121$ & $-0,034$ & 0,008 & $-0,009$ \\
\hline UBES & & & 1 & $0,246^{\star}$ & 0,144 & $0,232^{\star}$ & 0,149 & 0,146 & $0,192^{\star}$ & $0,222^{\star}$ & $0,200^{\star}$ & $0,213^{*}$ & $0,298^{\star}$ & $0,288^{\star}$ \\
\hline GOT & & & & 1 & $0,710^{\star *}$ & $0,535^{\star *}$ & $0,248^{*}$ & $-0,133$ & $-0,082$ & $-0,109$ & $-0,152$ & 0,117 & $0,258^{\star}$ & $0,215^{\star}$ \\
\hline GPT & & & & & 1 & $0,307^{\star}$ & 0,112 & $-0,070$ & $-0,059$ & $-0,067$ & $-0,084$ & $-0,016$ & 0,168 & 0,077 \\
\hline GGT & & & & & & 1 & $0,354^{\star}$ & $-0,122$ & $-0,122$ & 0,014 & $-0,102$ & 0,058 & 0,100 & 0,093 \\
\hline VCM & & & & & & & 1 & 0,014 & $-0,047$ & 0,102 & 0,037 & 0,139 & 0,073 & 0,133 \\
\hline $\begin{array}{l}\text { BIS11 } \\
\text { C }\end{array}$ & & & & & & & & 1 & $0,606^{\star \star}$ & $0,562^{\star *}$ & $0,865^{\star *}$ & $0,404^{\star \star}$ & $0,194^{*}$ & $0,341^{*}$ \\
\hline $\begin{array}{l}\text { BIS11 } \\
\text { M }\end{array}$ & & & & & & & & & 1 & $0,585^{\star *}$ & $0,824^{\star *}$ & $0,338^{*}$ & $0,234^{\star}$ & $0,318^{*}$ \\
\hline $\begin{array}{l}\text { BIS11 } \\
\mathrm{N}\end{array}$ & & & & & & & & & & 1 & $0,846^{\star *}$ & $0,252^{*}$ & $0,219^{\star}$ & $0,271^{\star}$ \\
\hline $\begin{array}{l}\text { BIS } 11 \\
\text { T }\end{array}$ & & & & & & & & & & & 1 & $0,388^{\star \star}$ & $0,247^{\star}$ & $0,361^{*}$ \\
\hline $\begin{array}{l}\text { OCDS } \\
0\end{array}$ & & & & & & & & & & & & 1 & $0,510^{\star *}$ & $0,884^{\star \star}$ \\
\hline $\begin{array}{l}\text { OCDS } \\
\mathrm{C}\end{array}$ & & & & & & & & & & & & & 1 & $0,849^{\star \star}$ \\
\hline $\begin{array}{l}\text { OCDS } \\
T\end{array}$ & & & & & & & & & & & & & & 1 \\
\hline
\end{tabular}

Nota. Años: Años de dependencia al alcohol; \%: Porcentaje de vida con dependencia al alcohol; UBE: Unidad de Bebida Estandar; BIS: Barratt Impulsiveness Scale (C: cognitivo, M: motor, N: no planificado, T: total); OCDS: Obsessive Compulsive Drinking Scale (O: obsesivo, C: Compulsivo, T: Total); GOT: Glutamano Oxalacetato Transaminasa; GPT: Glutamano Piruvato Transaminasa; GGT: Gamma Glutamil Transferasa; VCM: Volumen Corpuscular Medio.

${ }^{*} p<0,05 ;{ }^{* *} p<0,0001$ 
que reflejan los resultados en los últimos 20 ensayos de la prueba así lo acreditan, en la Tabla 3 se puede apreciar que el grupo control en estas variables del IGT (IGT 5 NET $\mathrm{AD}$, esta variable recoge el número de respuestas correctas dadas en los últimos 20 intentos, e IGT 5 NET DIS, esta variable mide justo lo contrario que la anterior) obtiene resultados próximos a una elección donde no ha habido el aprendizaje necesario para realizar correctamente la prueba. Si el grupo control obtiene malos resultados sólo puede haber diferencias significativas si los pacientes obtienen resultados catastróficos, este puede ser el caso en pacientes con daño cerebral severo o en el rango de la demencia, pero no es el caso de los pacientes que han participado en este estudio. Hay que recordar que se trata de pacientes ambulatorios no institucionalizados y con suficiente capacidad cognitiva como para firmar un consentimiento informado y participar en el estudio. El protocolo de valoración de estudio se realizó cuando esta prueba todavía no había sido cuestionada por la investigación más reciente y por ello fue incluida.

Con respecto a la otra prueba que no alcanza resultados significativos en la comparación entre los pacientes y los controles, el IAT, en el estudio de correlaciones con las variables clínicas (Tabla 4) se obtienen resultados que permiten explicar esta ausencia de significancia. EL IAT mide la preferencia automática e implícita de una persona hacia una categoría, en este estudio las bebidas alcohólicas. Cuando existe esta preferencia automática los tiempos de latencia a la hora de emparejar palabras relacionadas con la categoría a estudio con palabras con valencia positiva o negativa se modifica con respecto a una respuesta neutra. En este estudio se esperaba observar una latencia que indicase una preferencia de los pacientes frente a los controles por las bebidas alcohólicas, algo que inicialmente no se observó. Sin embargo, si se observan diferencias significativas en el grupo de pacientes cuando se tiene en cuenta el porcentaje de años de dependencia al alcohol, el OCDS total, el BIS no planificado y el BIS total. Estos resultados indican que el IAT es discriminativo cuando el craving de alcohol de los pacientes al alcohol está consolidado, es intenso y se relaciona con la impulsividad. $\mathrm{El}$ alcohol es una sustancia psicoactiva con poca potencia adictiva si la comparamos con el tabaco, la cocaína o los derivados mórficos. Nuestro grupo de pacientes presenta una dependencia al alcohol moderada, con un consumo de unos 90 gramos de etanol al día y con unos 13 años de media de dependencia al alcohol, con desviaciones estándar importantes, tal y como se puede apreciar en la Tabla 1. Es decir, no se trata de un grupo con una dependencia y craving al alcohol extremos, lo cual vuelve la muestra más heterogénea y hace que en un porcentaje importante de los pacientes el IAT no arroje resultados significativos. En resumen, los resultados parecen indicar que el IAT es una prueba discriminativa en aquellos pacientes con una dependencia y un craving al alcohol intensos y prolongados, y que se acompañan de una importante impulsividad.

También es importante para el personal sanitario que a diario trabaja en la desintoxicación y deshabituación alcohólica, conocer que variables relacionadas con el consumo de alcohol tienen una relación significativa con el deterioro cognitivo producido por el ARBD. Estas variables, de fácil recogida en las entrevistas diagnósticas iniciales, actuarían como marcadores de riesgo cuya presencia indicaría la necesidad de realizar un análisis neuropsicológico más exhaustivo. Las UBEs consumidas a diario durante el último mes es un marcador de consumo reciente, al igual que los parámetros que miden en la analítica sanguínea las consecuencias negativas del consumo abusivo de alcohol (GOT, GPT, GGT y VCM). De estas últimas, las transaminasas hepáticas (GOT, GPT y GGT) se relacionan con el consumo alcohólico realizado el mes previo, coincidiendo por lo tanto con las UBEs, mientras que el parámetro relacionado con los hematíes, el VCM, se relaciona, con menor especificidad que la GGT y las UBEs, con el consumo alcohólico realizado los tres meses anteriores a la valoración (Niemela, 2016). Tal y como indica el estudio de correlaciones realizado (Tabla 4), estos marcadores de consumo reciente, tienen una escasa relación con el funcionamiento cognitivo de los pacientes, y sólo parecen relacionarse débilmente con la prueba de aritmética.

Sin embargo, las variables relacionadas con el consumo de alcohol a largo plazo (años de dependencia al alcohol, porcentaje de vida de dependencia al alcohol, OCDS y BIS11) se relacionan de una forma más significativa con la función cognitiva. A más tiempo de dependencia al alcohol se observa un empeoramiento en la atención, en la memoria anterógrada, en la velocidad de procesamiento y el razonamiento abstracto. Puntuaciones más elevadas en el OCDS, que indican la presencia de un craving de alcohol más intenso y consolidado, se relacionan con un empeoramiento en la velocidad de procesamiento, la fluidez verbal y en el razonamiento abstracto y la flexibilidad cognitiva. Puntuaciones más elevadas en el BIS-11, que indican la presencia de una mayor impulsividad, se asocian especialmente con un empeoramiento de la función ejecutiva (fluidez verbal, resistencia a las interferencias, atención dividida, razonamiento abstracto y flexibilidad cognitiva) y con la memoria a corta plazo.

Los resultados obtenidos en la matriz de correlaciones con respecto al BIS-11 son muy significativos, destacando su alta correlación con un peor funcionamiento ejecutivo. Es necesario recordar que esta prueba mide la impulsividad de forma global, es decir no diferencia la impulsividad que pueda haber provocado el deterioro cognitivo asociado al consumo de alcohol frente a la impulsividad que pudiesen presentar previamente los pacientes y que contribuyó a que desarrollasen una dependencia al alcohol. Existen evidencias previas que confirman que ambos 
escenarios son posibles y compatibles. Los pacientes con más dependencia al alcohol y que inician su consumo problemático más precozmente presentan a nivel individual y familiar una mayor tendencia a la impulsividad (Bernstein et al., 2015; Jakubczyk et al., 2013) y en este estudio los pacientes presentan de forma significativa una mayor tendencia familiar al consumo problemático de alcohol (Tabla 2).

Lo que sí está claro en el presente estudio es que el BIS11 correlaciona especialmente con el OCDS (Tabla 5), indicando una estrecha relación entre la impulsividad y el craving de alcohol. Y estas dos dimensiones psicopatológicas son las que más se relacionan con el deterioro cognitivo en este estudio (Tabla 4). Lo más probable es que esta relación sea bidireccional, a más impulsividad y craving más consumo de alcohol y por lo tanto más deterioro cognitivo, y a más deterioro cognitivo peor función ejecutiva y por lo tanto más impulsividad y craving de alcohol.

Estos datos confirman investigaciones previas que indican que la intensidad del deterioro cognitivo asociado al consumo de alcohol vendrá determinada por el historial de consumo de alcohol a lo largo de la vida, y no por el consumo más reciente, por muy intenso que haya sido (Hayes et al., 2016; Horton et al., 2015).

Por lo tanto, los resultados del presente estudio indican que los pacientes con trastorno por uso de alcohol presentan, frente a los controles sanos, una peor capacidad de planificación y una menor flexibilidad cognitiva, y a esto se suman alteraciones atencionales y en la memoria anterógrada. Estas alteraciones claramente perjudicarían a los pacientes a la hora de seguir un programa de deshabituación alcohólica planificado, en el cual hubiera que adherirse a una pauta psicofarmacológica y a una intervención psicoterapéutica reglada. Además, estas alteraciones cognitivas favorecerían las recaídas en el consumo de alcohol. Así pues, los resultados de este estudio confirman los hallazgos de la investigación previa (Evren, Durkaya, Evren, Dalbudak y Cetin, 2012; Romero-Martinez et al., 2020). Es importante recordar, y así se confirma en el presente estudio, en el cual no se encontró ningún punto de corte para ninguna prueba cognitiva que permitiese diferenciar a un nivel individual a los pacientes de los controles sanos, que el deterioro cognitivo asociado al consumo de alcohol es dimensional y, por lo tanto, el deterioro que pueda presentar cada paciente será variable. Este deterioro puede intuirse a través del historial de consumo obtenido en la entrevista clínica inicial, pero sólo podrá conocerse con claridad la intensidad del mismo y sus posibles repercusiones pronósticas realizando una batería de pruebas cognitivas sistematizada.

Existen limitaciones en el presente estudio que deben ser indicadas. La principal es la naturaleza transversal del diseño. Esta transversalidad no permite aclarar con exactitud la relación entre la impulsividad medida con el BIS-
11 y el trastorno por uso de alcohol, ni determinar la influencia en la evolución y el pronóstico de las alteraciones cognitivas detectadas. Otro problema asociado al diseño transversal es la dificultad para medir retrospectivamente el consumo de alcohol con el máximo detalle. Pacientes con una historia de trastorno por uso de alcohol de igual duración pueden presentar un deterioro cognitivo producido por el consumo distinto en función de sus patrones de consumo durante el mismo. Dada la heterogeneidad de los pacientes en lo que a su historial de consumo de alcohol se refiere (Tabla 2), un tamaño muestral más grande hubiese permitido confirmar con más intensidad los resultados obtenidos. Finalmente, los criterios de inclusión / exclusión utilizados en este estudio determinan que los pacientes con trastorno por uso de alcohol de baja gravedad hayan sido excluidos, por ello, las conclusiones de este estudio sólo son aplicables a pacientes con trastorno por uso de alcohol moderado o grave.

A pesar de estas limitaciones, podemos afirmar, que el presente estudio confirma la presencia de deterioro cognitivo en los pacientes con trastorno por uso de alcohol moderado o grave que inician un tratamiento ambulatorio de deshabituación alcohólica. Este deterioro implica en estos pacientes un daño cognitivo que afecta a su capacidad atencional, memoria anterógrada y función cognitiva poniendo en riesgo, en función de la intensidad del deterioro cognitivo que presente cada paciente, sus posibilidades de alcanzar la abstinencia y consolidarla evitando la recaída. El deterioro cognitivo en el presente estudio se relaciona con el tiempo de dependencia, y no con el consumo reciente, y con la presencia de impulsividad. Además, estos dos factores determinan la presencia de una actitud implícita hacia las bebidas alcohólicas más favorable, lo cual también implica un riesgo de recaída más elevado. Dada la heterogeneidad en la historia de consumo que presentan los pacientes con trastorno por uso de alcohol que inician tratamiento ambulatorio es recomendable valorar la presencia de deterioro cognitivo de forma individual con una batería de pruebas cognitivas sistematizada.

\section{Reconocimientos}

Este estudio recibió apoyo de la Delegación del Gobierno para el Plan Nacional sobre Drogas, de la Secretaría de Estado de Servicios Sociales e Igualdad del Ministerio de Sanidad y Consumo (Número de Expediente: 2016I070). Los autores tambien agradecen el apoyo del Gobierno del Principado de Asturias PCTI-2018-2022 IDI/2018/235.

\section{Conflicto de intereses}

Los autores declaran la ausencia de conflictos de intereses en relación al estudio, su autoría, y / o a la publicación de este manuscrito. 


\section{Referencias}

Aharonovich, E., Campbell, A. N. C., Shulman, M., Hu, M. C., Kyle, T., Winhusen, T. y Nunes, E. V. (2018). Neurocognitive Profiling of Adult Treatment Seekers Enrolled in a Clinical Trial of a Web-delivered Intervention for Substance Use Disorders. Journal of Addiction Medicine, 12, 99-106. doi:10.1097/adm.0000000000000372.

Anton, R. F. (2000). Obsessive-compulsive aspects of craving: Development of the Obsessive Compulsive Drinking Scale. Addiction, 95 (Suppl. 2), 211-217.

Bech, P. (1990). Psychometric developments of the Hamilton scales: the spectrum of depression, dysthymia, and anxiety. Psychopharmacology Series, 9, 72-79.

Bernstein, M. H., McSheffrey, S. N., van den Berg, J. J., Vela, J. E., Stein, L. A., Roberts, M. B., ... Clarke, J. G. (2015). The association between impulsivity and alco$\mathrm{hol} /$ drug use among prison inmates. Addictive Behaviors, 42, 140-143. doi:10.1016/j.addbeh.2014.11.016.

Briere, F. N., Rohde, P., Seeley, J. R., Klein, D. y Lewinsohn, P. M. (2014). Comorbidity between major depression and alcohol use disorder from adolescence to adulthood. Comprehensive Psychiatry, 55, 526-533. doi:10.1016/j.comppsych.2013.10.007.

Carmona-Perera, M., Sumarroca-Hernandez, X., Santolaria-Rossell, A., Perez-Garcia, M. y Reyes Del Paso, G. A. (2019). Blunted autonomic responses to emotional stimuli in alcoholism: relevance of impulsivity. Adicciones, 31, 221-232. doi:10.20882/adicciones.1146.

del Ser Quijano, T., Sanchez Sanchez, F., Garcia de Yebenes, M. J., Otero Puime, A., Zunzunegui, M. V. y Munoz, D. G. (2004). Spanish version of the 7 Minute screening neurocognitive battery. Normative data of an elderly population sample over 70. Neurologia, 19, 344-358.

Draper, B., Karmel, R., Gibson, D., Peut, A. y Anderson, P. (2011). Alcohol-related cognitive impairment in New South Wales hospital patients aged 50 years and over. Australian and New Zealand Journal of Psychiatry, 45, 985992. doi:10.3109/00048674.2011.610297.

Elwood, R. W. (1995). The California Verbal Learning Test: Psychometric characteristics and clinical application. Neuropsychology Review, 5, 173-201.

Erdozain, A. M., Morentin, B., Bedford, L., King, E., Tooth, D., Brewer, C., ... Carter, W. G. (2014). Alcohol-related brain damage in humans. PloS One, 9, e93586. doi:10.1371/journal.pone.0093586.

Evren, C., Durkaya, M., Evren, B., Dalbudak, E. y Cetin, R. (2012). Relationship of relapse with impulsivity, novelty seeking and craving in male alcohol-dependent inpatients. Drug and Alcohol Review, 31, 81-90. doi:10.1111/ j.1465-3362.2011.00303.x.

Florez, G., Espandian, A., Villa, R. y Saiz, P. A. (2019). Clinical implications of cognitive impairment and alcohol dependence. Adicciones, 31, 3-7. doi:10.20882/adicciones. 1284 .
Galvin, R., Brathen, G., Ivashynka, A., Hillbom, M., Tanasescu, R. y Leone, M. A. (2010). EFNS guidelines for diagnosis, therapy and prevention of Wernicke encephalopathy. European Journal of Neurology, 17, 14081418. doi:10.1111/j.1468-1331.2010.03153.x.

Golpe, S., Isorna, M., Barreiro, C., Brana, T. y Rial, A. (2017). Binge drinking among adolescents: Prevalence, risk practices and related variables. Adicciones, 29, 256267. doi:10.20882/adicciones.932.

Hagen, E., Erga, A. H., Hagen, K. P., Nesvag, S. M., McKay, J. R., Lundervold, A. J. y Walderhaug, E. (2016). Assessment of executive function in patients with substance use disorder: A comparison of inventory- and performance-based assessment. Journal of Substance Abuse Treatment, 66, 1-8. doi:10.1016/j.jsat.2016.02.010.

Hayes, V., Demirkol, A., Ridley, N., Withall, A. y Draper, B. (2016). Alcohol-related cognitive impairment: current trends and future perspectives. Neurodegenerative Disease Management, 6, 509-523. doi:10.2217/nmt-2016-0030.

Horton, L., Duffy, T., Hollins Martin, C. y Martin, C. R. (2015). Comprehensive assessment of alcohol-related brain damage (ARBD): Gap or chasm in the evidence? Journal of Psychiatric and Mental Health Nursing, 22, 3-14. doi:10.1111/jpm.12156.

Jakubczyk, A., Klimkiewicz, A., Mika, K., Bugaj, M., Konopa, A., Podgorska, A., ... Wojnar, M. (2013). Psychosocial predictors of impulsivity in alcohol-dependent patients. Journal of Nervous and Mental Disease, 201, 43-47. doi:10.1097/NMD.0b013e31827aaf9d.

Koob, G. F. (2003). Alcoholism: Allostasis and beyond. Alcoholism, Clinical and Experimental Research, 27, 232-243. doi:10.1097/01.alc.0000057122.36127.c2.

Koob, G. F. y Volkow, N. D. (2010). Neurocircuitry of addiction. Neuropsychopharmacology, 35, 217-238. doi:10.1038/ npp.2009.110.

Laramee, P., Leonard, S., Buchanan-Hughes, A., Warnakula, S., Daeppen, J. B. y Rehm, J. (2015). Risk of all-cause mortality in alcohol-dependent individuals: A systematic literature review and meta-analysis. EBioMedicine, 2, 1394-1404. doi:10.1016/j.ebiom.2015.08.040.

Litten, R. Z., Ryan, M. L., Falk, D. E., Reilly, M., Fertig, J. B. y Koob, G. F. (2015). Heterogeneity of alcohol use disorder: Understanding mechanisms to advance personalized treatment. Alcoholism, Clinical and Experimental Research, 39, 579-584. doi:10.1111/acer.12669.

Maharasingam, M., Macniven, J. A. y Mason, O. J. (2013). Executive functioning in chronic alcoholism and Korsakoff syndrome. Journal of Clinical and Experimental Neuropsychology, 35, 501-508. doi:10.1080/13803395.2013.795527.

Moretti, R., Caruso, P., Dal Ben, M., Gazzin, S. y Tiribelli, C. (2017). Thiamine and alcohol for brain pathology: Super-imposing or different causative factors for brain damage? Current Drug Abuse Reviews, 10, 44-51. doi:10.21 74/1874473711666180402142012. 
Mujica-Parodi, L. R., Carlson, J. M., Cha, J. y Rubin, D. (2014). The fine line between 'brave' and 'reckless': amygdala reactivity and regulation predict recognition of risk. Neuroimage, 103, 1-9. doi:10.1016/j.neuroimage.2014.08.038.

Niemela, O. (2016). Biomarker-based approaches for assessing alcohol use disorders. International Journal of Environmental Research and Public Health, 13, 166. doi:10.3390/ijerph13020166.

Nyhus, E. y Barcelo, F. (2009). The Wisconsin Card Sorting Test and the cognitive assessment of prefrontal executive functions: A critical update. Brain and Cognition, 71, 437-451. doi:10.1016/j.bandc.2009.03.005.

Ostafin, B. D., Marlatt, G. A. y Greenwald, A. G. (2008). Drinking without thinking: An implicit measure of alcohol motivation predicts failure to control alcohol use. Behaviour Research and Therapy, 46, 1210-1219. doi:10.1016/j.brat.2008.08.003.

Patton, J. H., Stanford, M. S. y Barratt, E. S. (1995). Factor structure of the Barratt impulsiveness scale. Journal of Clinical Psychology, 51, 768-774.

Rehm, J., Rehm, M. X., Shield, K. D., Gmel, G. y Gual, A. (2013). Alcohol consumption, alcohol dependence and related harms in Spain, and the effect of treatment-based interventions on alcohol dependence. Adicciones, 25, 11-18.

Rehm, J., Shield, K. D., Gmel, G., Rehm, M. X. y Frick, U. (2013). Modeling the impact of alcohol dependence on mortality burden and the effect of available treatment interventions in the European Union. European Neuropsychopharmacology, 23, 89-97. doi:10.1016/j.euroneuro.2012.08.001.

Rehm, J., Allamani, A., Aubin, H. J., Della Vedova, R., Elekes, Z., Frick, U., ... Wojnar, M. (2015a). People with alcohol use disorders in specialized care in eight different European countries. Alcohol and Alcoholism, 50, 310-318. doi:10.1093/alcalc/agv009.

Rehm, J., Allamani, A., Della Vedova, R., Elekes, Z., Jakubczyk, A., Landsmane, I., ... Wojnar, M. (2015b). General practitioners recognizing alcohol dependence: a large cross-sectional study in 6 European countries. Annals of Family Medicine, 13, 28-32. doi:10.1370/afm.1742.

Rickham, P. P. (1964). Human Expermentation, Code of Ethics of the World Medical Association. Declaration of Hensinki. British Medical Journal, 2, 177.

Ridley, N. J., Draper, B. y Withall, A. (2013). Alcohol-related dementia: An update of the evidence. Alzheimer's Research $\mathcal{E}$ Therapy, 5, 3. doi:10.1186/alzrt157.

Romero-Martinez, A., Vitoria-Estruch, S. y Moya-Albiol, L. (2020). Cognitive profile of long-term abstinent alcoholics in comparison with non-alcoholics. Adicciones, 32, 19-31. doi:10.20882/adicciones.1079.

Sachdeva, A., Chandra, M., Choudhary, M., Dayal, P. y Anand, K. S. (2016). Alcohol-related dementia and neu- rocognitive impairment: A review study. International Journal of High Risk Behaviors Eं Addictions, 5, e27976. doi:10.5812/ijhrba.27976.

Scarpina, F. y Tagini, S. (2017). The Stroop Color and Word Test. Frontiers in Psychology, 8, 557. doi:10.3389/ fpsyg.2017.00557.

Shoval, G., Shmulewitz, D., Wall, M. M., Aharonovich, E., Spivak, B., Weizman, A. y Hasin, D. (2014). Alcohol dependence and suicide-related ideation/behaviors in an Israeli household sample, with and without major depression. Alcoholism, Clinical and Experimental Research, 38, 820-825. doi:10.1111/acer.12290.

Stavro, K., Pelletier, J. y Potvin, S. (2013). Widespread and sustained cognitive deficits in alcoholism: A meta-analysis. Addiction Biology, 18, 203-213. doi:10.1111/j.13691600.2011.00418.x.

Steinborn, M. B., Langner, R., Flehmig, H. C. y Huestegge, L. (2018). Methodology of performance scoring in the d2 sustained-attention test: Cumulative-reliability functions and practical guidelines. Psychological Assessment, 30, 339-357. doi:10.1037/pas0000482.

Steingroever, H., Wetzels, R., Horstmann, A., Neumann, J. y Wagenmakers, E. J. (2013). Performance of healthy participants on the Iowa Gambling Task. Psycholological Assessment, 25, 180-193. doi:10.1037/a0029929.

Volkow, N. D., Koob, G. F. y McLellan, A. T. (2016). Neurobiologic advances from the brain disease model of addiction. New England Journal of Medicine, 374, 363-371. doi:10.1056/NEJMra1511480.

Wollenweber, F. A., Halfter, S., Brugmann, E., Weinberg, C., Cieslik, E. C., Muller, V. I., ... Eickhoff, S. B. (2014). Subtle cognitive deficits in severe alcohol addicts-do they show a specific profile? Journal of Neuropsychology, 8, 147-153. doi:10.1111/jnp.12001.

Zahr, N. M. y Pfefferbaum, A. (2017). Alcohol's effects on the brain: Neuroimaging results in humans and animal models. Alcohol Research: Current Reviews, 38, 183-206. 
